\title{
Acute Macular Neuroretinopathy in a 15-Year-Old Boy: Optical Coherence Tomography and Visual Acuity Findings
}

\author{
Shinji Makino Hironobu Tampo \\ Department of Ophthalmology, Jichi Medical University, Shimotsuke, Japan
}

\section{Key Words}

Acute macular neuroretinopathy · Optical coherence tomography · Photoreceptor inner segment/outer segment junction · Retinal pigment epithelium

\begin{abstract}
Purpose: To report a case of acute macular neuroretinopathy (AMN) in a 15-year-old boy. Methods: Images were obtained with fundus photography and optical coherence tomography (OCT). Results: The patient complained of blurred vision and a small central scotoma in the left eye. Left visual acuity was 0.3 . Fundus photographs revealed a small dark area in the fovea of the left eye. OCT showed attenuation of the photoreceptor inner segment (IS)/outer segment (OS) line and the OS/retinal pigment epithelium (RPE) line in the left eye. One week after the initial visit, left visual acuity had improved to 0.6 , and there was partial recovery of the IS/OS line with focal thinning of the OS/RPE line. One month later, left visual acuity had improved to 0.8 , and OCT showed complete restoration of the IS/OS line and the OS/RPE line. Conclusions: To our knowledge, this is the youngest patient reported with a diagnosis of AMN. Changes in microstructural findings and visual acuity were analogous during recovery of $\mathrm{AMN}$ in our patient.

(c) 2014 S. Karger AG, Basel
\end{abstract}

\section{Introduction}

Acute macular neuroretinopathy (AMN) is a rare condition that produces transient or permanent visual impairment [1, 2]. In the original description of AMN, Bos and Deutman [1] proposed that the characteristic red wedge-shaped paracentral lesions of AMN involved the inner layers of the macula. Optical coherence tomography (OCT) is currently the best 
Makino et al.: Acute Macular Neuroretinopathy in a 15-Year-Old Boy: Optical Coherence Tomography and Visual Acuity Findings

method to visualize structural changes that occur during disease activity. Using OCT, several investigators have proved that the structural abnormalities in AMN are confined to the outer retina [3-14], and this clinical entity has been renamed 'acute macular outer retinopathy' [3, 7]. More recently, Sarraf et al. [14] proposed a classification of AMN. According to their report, AMN lesions may occur above or below the outer plexiform layer (OPL). In addition, AMN occurs predominantly in young women [2]. To our knowledge, there are few reports in the literature of cases of AMN in teenagers $[2,10]$. Here, we describe OCT changes in a 15year-old boy with AMN.

\section{Case Report}

A 15-year-old Japanese boy presented with a 6-day history of acute painless blurred vision and a small central scotoma in the left eye. Best corrected visual acuity (BCVA) was 0.8 and 0.3 in the right and left eyes, respectively. Intraocular pressure was $16 \mathrm{~mm} \mathrm{Hg}$ in both eyes. The patient had no specific past medical history. No inflammatory cells were observed in the anterior segment or vitreous of either eye. Fundus examination revealed no specific abnormalities in the right eye (fig. 1a). In the left eye, there was a small dark area in the fovea (fig. 1b, fig. 2a). OCT (RS-3000; Nidek, Japan) revealed no specific abnormalities in the right eye; however, in the left eye, disruption of the photoreceptor inner segment (IS)/outer segment (OS) junction line and the OS/retinal pigment epithelium (RPE) line in the foveal area (fig. 2d, e) was found. The patient was monitored without treatment. After 1 week, BCVA had improved to 0.6 in the left eye and OCT revealed partial restoration of the IS/OS line with focal thinning of the OS/RPE line in the foveal area (fig. 2f, g). After 1 month, the patient's complaints had resolved and BCVA had improved to 0.8 in the left eye. OCT showed complete restoration of the IS/OS line and the OS/RPE line (fig. $2 \mathrm{~h}$, i). Fluorescein angiography, indocyanine green angiography, and multifocal electroretinogram were not available in this case.

\section{Discussion}

AMN is different from the many clinical diseases affecting the central fundus, such as central serous chorioretinopathy, acute posterior multifocal pigment epitheliopathy, acute retinal pigment epithelitis, serpiginous choroiditis, presumed ocular histoplasmosis syndrome, multiple evanescent white dots syndrome, and acute zonal occult outer retinopathy (AZOOR) complex $[1,2,12]$. In this patient, we initially suspected the disease to be AZOOR. However, in brief, fundus photographs revealed a small dark area in the fovea, and OCT showed attenuation of the IS/OS line and the OS/RPE line rather than inner retinal layer abnormality. Therefore, we considered that these findings were compatible with AMN.

Turbeville et al. [2] described 41 cases reported in the English-language medical literature from 1975 to 2002. According to their review, AMN is seen more frequently in female than male patients; of their 41 cases, 34 (83\%) were found in female patients with an average age of 27.9 years (range 17-53). Further, considering male teenage patients, the 41 patients included two 17-year-old male patients and one 18-year-old male patient. Each of these 3 patients had experienced a previous event before presentation, including flu-like symptoms, administration of epinephrine for allergic food reaction, and chest trauma. In 2012, Rifkin and Schaal [10] described a 16-year-old girl with AMN after a flu-like illness. According to further literature searches and our knowledge, ours is the youngest male 
Makino et al.: Acute Macular Neuroretinopathy in a 15-Year-Old Boy: Optical Coherence Tomography and Visual Acuity Findings

patient with a diagnosis of AMN and no previous event. We recommend that AMN be considered in young male patients with acute onset of central or paracentral scotoma.

The causes of AMN are unknown, but numerous factors have been reported in association with its onset. These factors include oral contraceptives, flu-like illness, contrast media, epinephrine, trauma, a history of headache or migraine headache, postpartum hypotension, and hypotensive shock [2]. More recently, Sarraf et al. [14] proposed that the pathogenesis of AMN involves occlusion of either the superficial capillary plexus or the deep capillary plexus located in the innermost and outermost portion of the inner nuclear layer (INL). Although our patient had no predisposing event, we speculated that a transient ischemic episode might have contributed to the retinal changes seen in this case.

Several detailed reports on OCT findings in AMN patients describe structural changes, including IS/OS and OS/RPE loss and outer nuclear layer (ONL) thinning [3-14]. In addition, acute involvement of the ONL and OPL manifests as OCT hyperreflectivity $[6,8,9,11,13]$. The hallmark long-term change is ONL thinning regardless of whether the IS/OS defects resolve in the recovery phase [9, 11-14]. More recently, Sarraf et al. [14] proposed a classification of AMN. According to their report, 2 types of AMN lesions may occur - above or below the OPL. Type 1 is characterized by hyperreflective bands in the OPL/INL region with subsequent INL thinning. Type 2 is distinguished by hyperreflective bands in the OPL/ONL region with subsequent ONL thinning. Our OCT findings were similar to those previously reported for other AMN patients [3-14] and corresponded to type 2 AMN proposed by Sarraf et al. [14]. However, ONL and OPL thinning were not detected because the lesion was limited to the foveal area in our case. Furthermore, our case showed no OPL and/or ONL hyperreflectivity because examination was only performed 6 days after the onset.

Our findings were based on a single case of AMN, and fluorescein angiography, indocyanine green angiography, and multifocal electroretinogram were not available. However, changes in the microstructural findings and visual acuity were analogous during recovery of AMN in our patient. Finally, OCT was useful in demonstrating not only acute loss of visualization of the IS/OS line but also focal thinning of the OS/RPE line in a patient with AMN.

\section{Disclosure Statement}

The authors have no conflicts of interest to disclose.

\section{References}

1 Bos PJ, Deutman AF: Acute macular neuroretinopathy. Am J Ophthalmol 1975;80:573-584.

$\checkmark 2$ Turbeville SD, Cowan LD, Gass JD: Acute macular neuroretinopathy: a review of the literature. Surv Ophthalmol 2003;48:1-11.

- Hughes EH, Siow YC, Hunyor AP: Acute macular neuroretinopathy: anatomic localisation of the lesion with high-resolution OCT. Eye (Lond) 2009;23:2132-2134.

4 Monson BK, Greenberg PB, Greenberg E, Fujimoto JG, Srinivasan VJ, Duker JS: High-speed, ultra-highresolution optical coherence tomography of acute macular neuroretinopathy. Br J Ophthalmol 2007;91:119-120.

-5 Neuhann IM, Inhoffen W, Koerner S, Bartz-Schmidt KU, Gelisken F: Visualization and follow-up of acute macular neuroretinopathy with the Spectralis HRA+OCT device. Graefes Arch Clin Exp Ophthalmol 2010;248:1041-1044.

6 Maschi C, Schneider-Lise B, Paoli V, Gastaud P: Acute macular neuroretinopathy: contribution of spectraldomain optical coherence tomography and multifocal ERG. Graefes Arch Clin Exp Ophthalmol 2011;249:827-831. 


\begin{tabular}{l|l}
\hline Case Rep Ophthalmol 2014;5:11-15 \\
\hline DOI: 10.1159/000358166 & $\begin{array}{l}\text { ○ 2014 S. Karger AG, Basel } \\
\text { www.karger.com/cop }\end{array}$ \\
\hline
\end{tabular}

Makino et al.: Acute Macular Neuroretinopathy in a 15-Year-Old Boy: Optical Coherence Tomography and Visual Acuity Findings

7 Yeh S, Hwang TS, Weleber RG, Watzke RC, Francis PJ: Acute macular outer retinopathy (AMOR): a reappraisal of acute macular neuroretinopathy using multimodality diagnostic testing. Arch Ophthalmol 2011;129:365-368.

8 Baumüller S, Holz FG: Early spectral-domain optical coherence tomography findings in acute macular neuroretinopathy. Retina 2012;32:409-410.

-9 Azar G, Wolff B, Cornut PL, Sahel JA, Mauget-Faÿsse M: Spectral domain optical coherence tomography evolutive features in acute macular neuroretinopathy. Eur J Ophthalmol 2012;22:850-852.

$>10$ Rifkin L, Schaal S: Teenage acute macular neuroretinopathy. Eur J Ophthalmol 2012;22:674-676.

11 Garg A, Shah AN, Richardson T, O'Sullivan E, Eleftheriadis H: Early features in acute macular neuroretinopathy. Int Ophthalmol 2013, Epub ahead of print.

12 Vance SK, Spaide RF, Freund KB, Wiznia R, Cooney MJ: Outer retinal abnormalities in acute macular neuroretinopathy. Retina 2011;31:441-445.

13 Fawzi AA, Pappuru RR, Sarraf D, Le PP, McCannel CA, Sobrin L, Goldstein DA, Honowitz S, Walsh AC, Sadda SR, Jampol LM, Eliott D: Acute macular neuroretinopathy: long-term insights revealed by multimodal imaging. Retina 2012;32:1500-1513.

14 Sarraf D, Rahimy E, Fawzi AA, Sohn E, Barbazetto I, Zacks DN, Mittra RA, Klancnik JM, Mrejen S, Goldberg NR, Beardsley R, Sorenson JA, Freund KB: Paracentral acute middle maculopathy: a new variant of acute macular neuroretinopathy associated with retinal capillary ischemia. JAMA Ophthalmol 2013;131:12751287.


Fig. 1. Fundus photographs at the initial visit in a 15-year-old boy with AMN. There are no specific abnormalities in the right eye (a). There is a slightly darker area in the fovea of the left eye (b). 
Case Reports in

Ophthalmology

\begin{tabular}{l|l}
\hline \multicolumn{2}{l}{ Case Rep Ophthalmol 2014;5:11-15 } \\
\hline DOI: $10.1159 / 000358166$ & $\begin{array}{l}\text { C 2014 S. Karger AG, Basel } \\
\text { www.karger.com/cop }\end{array}$ \\
\hline
\end{tabular}

Makino et al.: Acute Macular Neuroretinopathy in a 15-Year-Old Boy: Optical Coherence Tomography and Visual Acuity Findings
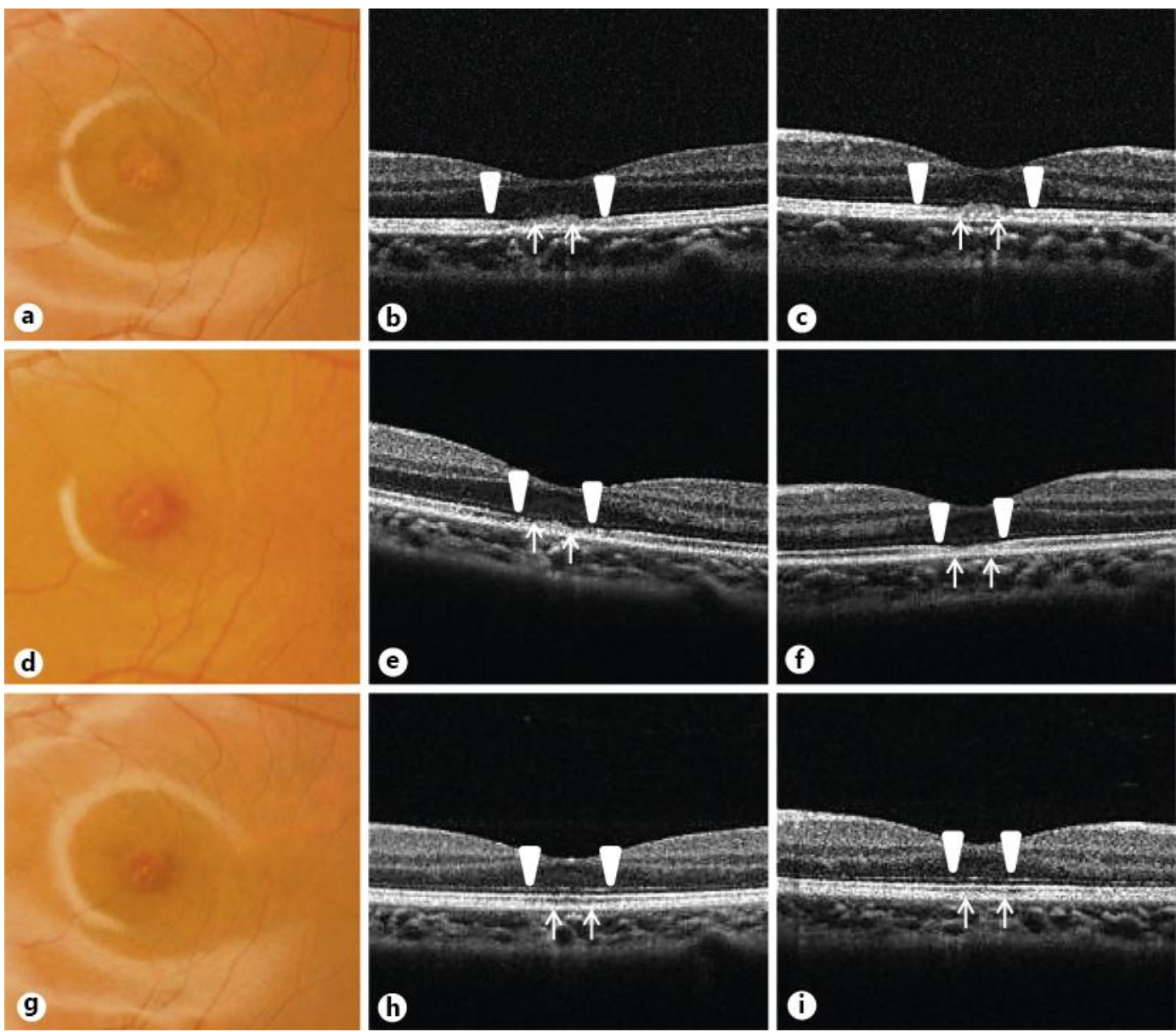

Fig. 2. High-magnification fundus photographs (a-c) and OCT images [d-i; horizontal $(\mathbf{d}, \mathbf{f}, \mathbf{h})$ and vertical $(\mathbf{e}, \mathbf{g}, \mathbf{i})$ scans] in a 15-year-old boy with AMN in the left eye. At the initial visit, there is a slightly darker area in the fovea (a) and the OCT images show attenuation of the IS/OS line and the OS/RPE line (d, e). After 1 week (b), OCT reveals partial restoration of the IS/OS line with focal thinning of the OS/RPE line (f, g). At 1 month (c), OCT shows complete restoration of the IS/OS line and the OS/RPE line (h, i). Arrowheads indicate the IS/OS line. Arrows indicate the OS/RPE line. 